\title{
Oligopoly with Hyperbolic Demand: A Differential Game Approach ${ }^{1}$
}

\author{
Luca Lambertini \\ Department of Economics, University of Bologna \\ Strada Maggiore 45, 40125 Bologna, Italy \\ Fax: +39-051-2092664; lamberti@spbo.unibo.it; \\ ENCORE, University of Amsterdam \\ Roetersstraat 11, WB1018 Amsterdam, The Netherlands; \\ and \\ Paul H. Nitze School of Advanced International Studies \\ Johns Hopkins University Bologna Center \\ Via Belmeloro, 11, 40126 Bologna, Italy
}

June 18, 2007

${ }^{1}$ I thank Davide Dragone, George Leitmann and Arsen Palestini for very insightful comments and suggestions. The usual disclaimer applies. 


\begin{abstract}
It is well known that the equilibrium solution of oligopoly games with isoelastic demand functions can be indeterminate. I revisit this issue through an open-loop differential game approach based on the assumption of sticky prices, to show that indeterminacy arises only in steady state, in the limit case where marginal costs tend to zero. Otherwise, at any time during the game, Pontryagin's Maximum Principle ensures the existence of a unique and well defined solution, irrespective of the size of marginal costs. Finally, I show that an analogous result holds in the feedback case, although the Bellman equation of the representative firm cannot be solved analytically.
\end{abstract}

JEL Codes: C73, D43, D92, L13

Keywords: optimal control, differential games, Cournot competition, sticky prices 


\section{Introduction}

Most of the existing literature on oligopoly theory (either static or dynamic) assumes linear demand functions, as this, in addition to simplifying calculations, also ensures both concavity and unicity of the equilibrium, which, in general, wouldn't be warranted in presence of convex demand systems (see Friedman, 1977; and Dixit, 1986, inter alia).

In particular, a classic textbook example of a market with convex demand is that where the demand function has a hyperbolic shape, this being a special case of a more general class of models based on isoelastic demand curves. As is well known, in such a case the maximum problem of a firm choosing the output level is indeterminate if marginal cost is nil, since the revenue generated by a hyperbolic (or, in general, isoelastic) demand is constant.

The aim of this paper is to illustrate a way out of the aforementioned problem, offered by dynamic game theory. To this purpose, I propose a differential oligopoly game where firms face implicit menu costs of adjusting outputs over time because market price is sticky (as in Simaan and Takayama, 1978; and Fershtman and Kamien, 1987, inter alia). The main results can be summarised as follows. Using the open-loop solution method, I prove that, for any given level of price stickiness, the first order condition on the individual firm's control yields a unique and well defined solution at any time during the game, as long as the co-state variable associated with the price dynamics differs from zero (which is necessary for Pontryagin's Maximum Principle to hold). Then, I show that

- the steady state is stable for any degree of price stickiness, provided that the number of firms in the market is at least equal to two;

- the monopoly equilibrium is stable, provided that the degree of price 
stickiness is below a critical threshols ensuring concavity; and

- indeterminacy arises at the steady state only, if the marginal production cost tends to zero.

The feedback equilibrium cannot be characterised analytically, as the game is not a linear quadratic one. However, the first order condition taken on the Bellman equation of the generic firm shows that a unique solution always exists at any time during the game, also in the limit case where marginal cost is equal to zero.

The remainder of the paper is structured as follows. Section 2 summarises the static game. The open-loop solution of the differential game with sticky market price is investigated in section 3. The feedback problem is briefly accounted for in section 4 . Section 5 contains some concluding remarks.

\section{A summary of the static game}

$N$ firms supply individual quantities $q_{i}, i=1,2,3, \ldots N$. The good is homogeneous, and market demand is $p=a / Q, Q=\sum_{i=1}^{N} q_{i}$. Production entails a total cost $c q_{i}^{2}$. Market competition takes place à la Cournot-Nash; therefore, firm $i$ chooses $q_{i}$ so as to maximise profits $\pi_{i}=\left(p-c q_{i}\right) q_{i}$. this entails that the following first order condition must be satisfied:

$$
\frac{\partial \pi_{i}}{\partial q_{i}}=\frac{a \sum_{j \neq} q_{j}}{\left(q_{i}+\sum_{j \neq} q_{j}\right)^{2}}-2 c q_{i}=0
$$

and the associated second order condition:

$$
\frac{\partial^{2} \pi_{i}}{\partial q_{i}^{2}}=-\frac{2 a \sum_{j \neq} q_{j}}{\left(q_{i}+\sum_{j \neq} q_{j}\right)^{3}}-2 c \leq 0
$$


which is always met. Imposing the symmetry condition $q_{i}=q$ for all $q_{i}=$ $1,2,3, \ldots N$, one obtains the Cournot-Nash equilibrium

$$
q^{C N}=\frac{1}{N} \sqrt{\frac{a(N-1)}{2 c}} ; p^{C N}=\frac{\sqrt{2 a c(N-1)}}{N-1}
$$

yielding profits $\pi^{C N}=a(N+1) /\left(2 N^{2}\right)$. Note that one must exclude the case $N=1$ as the above solution is indeterminate under monopoly. As we shall see in the remainder of the paper, this does not hold true in general, if an appropriate dynamics is adopted. If the $N$ firms were operating under perfect competition, then $p^{*}=2 c q_{i}$ and therefore $q^{*}=\sqrt{a /(2 c N)}$.

Moreover, it is apparent that the above solutions (i.e., both the CournotNash equilibrium and the perfectly competitive equilibrium) are determinate for all $c>0$, while they become indeterminate in correspondence of $c=0$.

\section{The dynamic setup}

Consider the following dynamic version of the oligopoly game examined in the previous section. At any $t \in[0, \infty)$, each of $N$ firms supplies quantity $q_{i}(t), i=1,2,3, \ldots N$, of the same homogeneous good, at an instantaneous cost $C_{i}(t)=c q_{i}^{2}(t)$, where $c \geq 0$ is a constant parameter measuring marginal production cost. At any instant, the notional market demand function is:

$$
\widehat{p}(t)=\frac{a}{Q(t)}, Q(t)=\sum_{i=1}^{N} q_{i}(t) ; a>c .
$$

However, the current market price $p(t)$ is sticky and, as in Simaan and Takayama (1978) and Fershtman and Kamien (1987), ${ }^{1}$ its dynamics is de-

\footnotetext{
${ }^{1}$ See also Fershtman and Kamien (1990), Tsutsui and Mino (1990) and Cellini and Lambertini (2004).
} 
scribed by the following differential equation:

$$
\dot{p}(t)=s[\widehat{p}(t)-p(t)]
$$

The above dynamics establishes that price $p(t)$ adjusts proportionately to the difference between the correct price level given by the notional demand function (4) and the current price, the instantaneous speed of adjustment being measured by the constant parameter $s \in[0, \infty) .^{2}$ Firms face implicit menu costs generated by the price stickiness, so that they never supply the correct quantities leading to the market clearing price $p(t)=\widehat{p}(t)$ (except at the steady state, of course).

The problem of firm $i$ is to choose output $q_{i}(t)$ so as to maximisize its own discounted profits:

$$
J_{i}(p(t), \mathbf{q}(t)) \triangleq \int_{0}^{\infty}\left[p(t)-c q_{i}(t)\right] q_{i}(t) e^{-\rho t} d t
$$

s.t. the price dynamics (5) and the initial condition $p(0)=p_{0} \cdot \mathbf{q}(t)$ is the vector of all firms' controls.

Given that the notional demand function is hyperbolic, the feedback solution through the Bellman equation cannot be pursued as the problem at hand is not in a linear-quadratic form. Therefore, I will confine my attention to the open-loop solution. The Hamiltonian of firm $i$ is:

$\mathcal{H}_{i}(p(t), \mathbf{q}(t))=e^{-\rho t}\left\{\left[p(t)-c q_{i}(t)\right] q_{i}(t)+\lambda_{i}(t) s\left[\frac{a}{q_{i}(t)+\sum_{j \neq} q_{j}(t)}-p(t)\right]\right\}$

where $\lambda_{i}(t)=\mu_{i}(t) e^{\rho t}$, and $\mu_{i}(t)$ is the co-state variable that firm $i$ associates to $p(t)$.

\footnotetext{
${ }^{2}$ Note that, if $s$ is infinitely high, then the price adjusts immediately to the notional level, which is equivalent to saying that price is not sticky at all.
} 
The necessary conditions are: ${ }^{3}$

$$
\begin{gathered}
\frac{\partial \mathcal{H}_{i}(\cdot)}{\partial q_{i}(t)}=p(t)-2 c q_{i}(t)-\frac{\lambda_{i}(t) a s}{\left[q_{i}(t)+\sum_{j \neq} q_{j}(t)\right]^{2}}=0 \\
-\frac{\partial \mathcal{H}_{i}(\cdot)}{\partial p(t)}=\dot{\lambda}_{i}(t)-\rho \lambda_{i}(t) \Leftrightarrow \\
\dot{\lambda}_{i}(t)=\lambda_{i}(t)(\rho+s)-q_{i}(t)
\end{gathered}
$$

with the transversality condition

$$
\lim _{t \rightarrow \infty} e^{-\rho t} \lambda_{i}(t) p(t)=0 .
$$

Now, solving (8) w.r.t. $\lambda_{i}(t)$, we obtain:

$$
\lambda_{i}(t)=\frac{\left[p(t)-2 c q_{i}(t)\right]\left[q_{i}(t)+\sum_{j \neq} q_{j}(t)\right]^{2}}{a s} .
$$

Before proceeding, it is worth noting that (8) and/or (11) imply that $p(t) \neq$ $2 c q_{i}(t)$ for all $s \in(0, \infty)$. Or, conversely:

Lemma 1 Provided $s \in(0, \infty)$ and $\lambda_{i}(t)>0$, the price will differ from marginal cost at any time $t$ during the game.

Additionally, and more importantly, (8) and/or (11) also involve: ${ }^{4}$

Proposition $2 \lambda_{i}(t)>0$ implies that $q_{i}(t), i=1,2,3, \ldots N$, is determined by the instantaneous (negatively sloped) best reply function

$$
q_{i}^{*}(t)=\frac{p(t)\left[\sqrt[3]{\varphi}-4 c \sum_{j \neq} q_{j}(t)\right]-p^{2}(t)-\left[\sqrt[3]{\varphi}+2 c \sum_{j \neq} q_{j}(t)\right]^{2}}{6 c \sqrt[3]{\varphi}} \in \mathbb{R}^{+}
$$

\footnotetext{
${ }^{3}$ Exponential discounting is omitted for brevity.

${ }^{4}$ The proof is omitted for brevity. However, note that equation (8) has three solutions w.r.t. $q_{i}(i)$, out of which only one - appearing in the text of Proposition 2 - is real and positive.
} 


$$
\begin{aligned}
\varphi= & 54 a c^{2} s \lambda_{i}(t)-\left[p(t)+2 c \sum_{j \neq} q_{j}(t)\right]^{3}+ \\
& 6 \sqrt{3 a c^{2} s \lambda_{i}(t)\left[27 a c^{2} s \lambda_{i}(t)-\left(p(t)+2 c \sum_{j \neq} q_{j}(t)\right)^{3}\right]}
\end{aligned}
$$

at all $t$ during the game, for all $c \in[0, a)$.

This result deserves to be spelled out in detail. The above Proposition establishes that, unlike what happens in the corresponding static game, in the present dynamic setup the optimal output of every single firm is determined as long as the co-state variable $\lambda_{i}(t)$ is not nil. Note that this fact, which is shown in equation (11), is also ensured by Pontryagin's Maximum Principle. Also note that Proposition 2 includes the degenerate case where marginal cost $c$ is equal to zero, in correspondence of which the solution of the static game is indeterminate.

The second order (sufficiency) condition for concavity requires:

$$
\frac{\partial^{2} \mathcal{H}_{i}(\cdot)}{\partial q_{i}^{2}(t)}=\frac{2 \lambda_{i}(t) a s}{\left[q_{i}(t)+\sum_{j \neq} q_{j}(t)\right]^{3}}-2 c \leq 0
$$

or, equivalently,

$$
\lambda_{i}(t) \leq \bar{\lambda}_{i}(t) \triangleq \frac{c\left[q_{i}(t)+\sum_{j \neq} q_{j}(t)\right]^{3}}{2 a s} .
$$

We can proceed to the characterisation of the control dynamics. Differentiating (8) w.r.t. time, we have:

$$
\dot{p}(t)-2 c \dot{q}_{i}(t)-\frac{\dot{\lambda}_{i}(t) a s}{\left[q_{i}(t)+\sum_{j \neq} q_{j}(t)\right]^{2}}+\frac{2 \lambda_{i}(t) a s\left[\dot{q}_{i}(t)+\sum_{j \neq} \dot{q}_{j}(t)\right]}{\left[q_{i}(t)+\sum_{j \neq} q_{j}(t)\right]^{3}}=0,
$$


that can be solved to obtain the following description of the dynamics of firm i's control: ${ }^{5}$

$$
\dot{q}_{i}=\frac{Q^{3} \dot{p}-a\left(\dot{\lambda}_{i} Q-2 \lambda \sum_{j \neq} \dot{q}_{j}\right) s}{2\left(c Q^{3} a s \lambda_{i}\right)}
$$

Imposing the symmetry conditions $\dot{q}_{j}=\dot{q}_{i}=\dot{q}$ and $q_{j}=q_{i}=q$, and then plugging (9) and (11) into (15), the dynamics of the optimal control can be rewritten as follows:

$$
\dot{q}=\frac{N^{2} q[p(2 s+\rho)-2 c q(s+\rho)]-a s(N+1)}{2 N^{2}(p-3 c q)} .
$$

Under the same symmetry conditions, the dynamics of price simplifies as follows:

$$
\dot{p}=s\left[\frac{a}{N q}-p(t)\right] \text {. }
$$

Imposing the stationarity condition $\dot{p}=0$, one obtains $p=a /(N q)$, which can be plugged into (16). At the steady state, $\dot{q}=0$ yields:

$$
q_{A}^{s s}=0 ; q_{B}^{s s}=\frac{1}{N} \sqrt{\frac{a[N(s+\rho)-s]}{2(s+\rho) c}}>0 ; q_{C}^{s s}=-\frac{1}{N} \sqrt{\frac{a[N(s+\rho)-s]}{2(s+\rho) c}}<0 .
$$

The solutions $q_{A}^{s s}$ and $q_{C}^{s s}$ can be disregarded for obvious reasons. In correspondence of $q^{s s}=q_{B}^{s s}$, the steady state price is:

$$
p^{s s}=a \sqrt{\frac{2(s+\rho) c}{a[N(s+\rho)-s]}}>2 c q^{s s} \forall s \in(0, \infty) .
$$

On the basis of (18-19), we have

Lemma 3 Provided $c>0$, the steady state equilibrium $\left(p^{s s}, q^{s s}\right)$ is economically acceptable for any $N \geq 1$ and any finite value of $s$.

\footnotetext{
${ }^{5}$ Henceforth, the indication of time is omitted for brevity.
} 
This is a key result of the dynamic analysis carried out so far: unlike the static model, where the equilibrium outcome (3) is acceptable only for $N \geq 2$, here the steady state of the dynamic setting is also well defined for the monopoly case, under some approapriate conditions on the relevant parameters.

At $\left(p^{s s}, q^{s s}\right)$, steady state profits amount to:

$$
\pi^{s s}=\frac{a[N(s+\rho)+s]}{2 N^{2}(s+\rho)} .
$$

The following result can be shown to hold:

Proposition 4 Take $c>0$. The steady state identified by

$$
p^{s s}=a \sqrt{\frac{2(s+\rho) c}{a[N(s+\rho)-s]}} ; q^{s s}=\frac{1}{N} \sqrt{\frac{a[N(s+\rho)-s]}{2(s+\rho) c}}
$$

is a saddle point for all $N \geq 3$. If $N \in\{1,2\}$, then:

- in $N=1,\left(p^{s s}, q^{s s}\right)$ is (i) a saddle point for all $s \in(0, \rho / 2)$; (ii) an unstable focus for all $s>\rho / 2$;

- in $N=2,\left(p^{s s}, q^{s s}\right)$ is (i) a saddle point for all $s \in(0,2 \rho)$; (ii) a stable node for all $s \in(2 \rho, 2.226 \rho)$; a stable focus for all $s>2.226 \rho$.

Proof. See the Appendix.

What the above Proposition establishes is not only that the open-loop solution of the dynamic game is determined (as opposed to the outcome of the corresponding static game), but also that the associated steady state point is always stable, provided that either (i) the number of firms is sufficiently high, or (ii) the speed of price adjustment is sufficiently low. These features deserve some additional comments. Property (i) indicates that competition 
(i.e., $N \geq 2$ ) ensures stability irrespective of the level of price stickiness. This is, per se, an element that cannot emerge from the corresponding static game. Conversely, in the monopoly case the steady state $\left(p^{s s}, q^{s s}\right)$ is unstable for all levels of $s$ above a critical threshold (and conversely). The reason for this result is to be found through the analysis of sufficiency condition (13).

In correspondence of $\left(p^{s s}, q^{s s}\right)$, the second order condition (13) revelas that

$$
\lambda_{i}(t) \leq \bar{\lambda}_{i}(t) \Leftrightarrow N^{2}(s+\rho)-N s-2 s \geq 0
$$

holding for all

$$
N \geq \widehat{N}=\frac{s \pm \sqrt{s^{2}+8 s(s+\rho)}}{2(s+\rho)},
$$

with $\widehat{N} \leq 2$ over the whole parameter set. Therefore, $N \geq 2$ suffices to ensure concavity. As a consequence,

Proposition $5\left(p^{s s}, q^{s s}\right)$ is surely a maximum point for all $N \geq 2$.

If instead $N=1$, then (13) is met for all $s<\rho / 2$ (i.e., in the region where $\left(p^{s s}, q^{s s}\right)$ is a saddle point), while it is violated elsewhere (in the region where $\left(p^{s s}, q^{s s}\right)$ is an unstable focus). Accordingly, there emerges that instability goes intuitively along with the lack of concavity at the monopoly equilibrium.

A simple comparative statics exercise can be carried out to assess the effect of an exogenous change in $s$ on the equilibrium levels of price, individual output and profits:

$$
\begin{aligned}
\frac{\partial p^{s s}}{\partial s} & =\frac{N a^{2} c \rho}{\sqrt{2 c N^{2}(s+\rho)[a(N(s+\rho)-s)]^{3}}}>0 \\
\frac{\partial q^{s s}}{\partial s} & =-\frac{N^{2} a c \rho}{2 \sqrt{2\left[c N^{2}(s+\rho)\right]^{3} a(N(s+\rho)-s)}}<0 \\
\frac{\partial \pi^{s s}}{\partial s} & =\frac{a \rho}{2 N^{2}(s+\rho)^{2}}>0
\end{aligned}
$$


This implies:

Corollary 6 For all $c>0$ and $N \geq 1$, an increase in $s$ entails a decrease in the steady state output (per firm as well as at the industry level) and a consequent increase in the steady state price. The balance of these two effects drives an increase in steady state profits.

Of course, this also drives a decrease in consumer surplus; therefore, one can conclude that price stickiness is good for firms while being bad for consumers (and, overall, for social welfare).

Then, taking the limit of $\left(p^{s s}, q^{s s}\right)$ as $s$ tends to infinity, it is easily ascertained that:

$$
\lim _{s \rightarrow \infty} p^{s s}=\lim _{\rho \rightarrow 0} p^{s s}=\frac{\sqrt{2 a c(N-1)}}{N-1} ; \lim _{s \rightarrow \infty} q^{s s}=\lim _{\rho \rightarrow 0} q^{s s}=\frac{1}{N} \sqrt{\frac{a(N-1)}{2 c}}
$$

and

$$
\lim _{s \rightarrow \infty} \pi^{s s}=\frac{a(N+1)}{2 N^{2}}
$$

Additionally:

$$
\begin{gathered}
\lim _{s \rightarrow 0} p^{s s}=\lim _{\rho \rightarrow \infty} p^{s s}=\frac{\sqrt{2 a c N}}{N} ; \lim _{s \rightarrow 0} q^{s s}=\lim _{\rho \rightarrow \infty} q^{s s}=\sqrt{\frac{a}{2 N c}} ; \\
\lim _{s \rightarrow 0} \pi^{s s}=\lim _{\rho \rightarrow \infty} \pi^{s s}=\frac{a}{2 N}
\end{gathered}
$$

corresponding to the perfectly competitive outcome of the static game.

These results prove the following additional Corollary to Proposition 3, describing the limit behaviour of the model, in correspondence of full price adjustment:

Corollary 7 In the limit, 
- as $s$ tends to infinity (or $\rho$ tends to zero), the steady state (which is a stable node) coincides with the static Cournot-Nash equilibrium, which is economically admissible for all $N \geq 2$;

- as $s$ tends to zero (or $\rho$ tends to infinity), the steady state (which is unstable) coincides with the static perfectly competitive equilibrium, which is admissible for all $N \geq 1$.

This limit result is broadly in accordance with the corresponding result obtained by Fershtman and Kamien (1987) in the model with linear demand and decreasing returns to scale.

Finally, observe that $q^{s s}$ becomes indeed indeterminate in the limit case where $c$ tends to zero, as it happens at the corresponding solution of the static game. This means that the steady state of the static game precisely portrays, in this special case, the outcome of the static setup. However, this is true only as soon as firms reach the steady state, while it is not true (as stated in Proposition 2) for an infinitely long time during the game.

\section{The feedback problem}

As stated above, the Bellman equation that would yield the feedback equilibrium of the game cannot be solved analytically since the game at hand is not a linear-quadratic one. However, a relevant implication of the first order condition can be easily drawn.

The Bellman equation for firm $i$ is:

$\rho V_{i}(p(t))=\max _{q_{i}(t)}\left\{\left[p(t)-c q_{i}(t)\right] q_{i}(t)+V_{i}^{\prime}(p(t)) s\left[\frac{a}{q_{i}(t)+\sum_{j \neq} q_{j}(t)}-p(t)\right]\right\}$ 
where $V_{i}(p)$ is the value function and $V_{i}^{\prime}(p)=\partial V_{i}(p(t)) / \partial p(t)$. Now, taking the first order condition, we have:

$$
p(t)-2 c q_{i}(t)-\frac{V_{i}^{\prime}(p(t)) a s}{\left[q_{i}(t)+\sum_{j \neq} q_{j}(t)\right]^{2}}=0,
$$

which admits a unique real solution w.r.t. $q_{i}(t)$, for any admissible value of the marginal cost, including $c=0$ :

$$
q_{i}^{*}(t)=\frac{p(t)\left[\sqrt[3]{\varpi}-4 c \sum_{j \neq} q_{j}(t)\right]-p^{2}(t)-\left[\sqrt[3]{\varpi}+2 c \sum_{j \neq} q_{j}(t)\right]^{2}}{6 c \sqrt[3]{\varpi}} \in \mathbb{R}^{+}
$$

where the expression for $\varpi$ corresponds to the expression for $\varphi$, except that $V_{i}^{\prime}(p(t))$ replaces $\lambda_{i}(t){ }^{6}$

\section{Concluding remarks}

I have revisited the Cournot oligopoly with isoelastic demand function using a dynamic approach based upon the assumption of price stickiness. This setup has allowed me to show that the indeterminacy issue commonly imputed to the static version of the game does not arise in general in the present dynamic reformulation, with the exception of the instant in which firms reach the steady state. In the open-loop case, at any other time during the game, the presence of co-state variables (which must differ from zero on the basis of the

\footnotetext{
${ }^{6}$ To this regard, it is appropriate to stress that $V_{i}^{\prime}(p(t))$ and $\lambda_{i}(t)$ are not equivalent, as shown by Caputo (2007): while $\lambda_{i}(t)$ can be considered a shadow price when looking at the open-loop solution, this is not true for $V_{i}^{\prime}(p(t))$, unless the game belongs to the class of perfect or state-redundant games producing strongly time consistent open-loop Nash equilibria. For a survey of perfect or state-redundant games, see Mehlmann (1988, ch. 4) and Docker et al. (2000, ch. 4).
} 
Maximum Principle) in the first order conditions ensure the existence of a well defined solution for firms' optimal outputs. Likewise, an analogous conclusion obtains in the feedback case, although the analytical solution remains of course out of reach since the game is not defined in a linear-quadratic form.

\section{Appendix}

Proof of Proposition 4 Using (9) and (11), the control dynamics (15) rewrites as follows:

$$
\dot{q}_{i}=\frac{a s\left(2 q_{i}+\sum_{j \neq i} q_{j}\right)+\left(q_{i}+\sum_{j \neq i} q_{j}\right)^{2}\left[p(2 s+\rho)-2 c(s+\rho) q_{i}\right]}{2\left(q_{i}+\sum_{j \neq i} q_{j}\right)\left[N\left(p-2 c q_{i}\right)-c\left(q_{i}+\sum_{j \neq i} q_{j}\right)\right]} .
$$

The above dynamics must be evaluated together with the state dynamics

$$
\dot{p}=s\left(\frac{a}{q_{i}+\sum_{j \neq i} q_{j}}-p\right) \text {. }
$$

The stability properties of this dynamic system can be assessed by evaluating the trace and determinant of the associated Jacobian matrix:

$$
J=\left[\begin{array}{ll}
\frac{\partial \dot{p}}{\partial p} & \frac{\partial \dot{p}}{\partial q_{i}} \\
\frac{\partial \dot{q}_{i}}{\partial p} & \frac{\partial \dot{q}_{i}}{\partial q_{i}}
\end{array}\right]
$$

At $\left(p^{s s}, q^{s s}\right)$, the trace and determinant of matrix $J$ are:

$$
\begin{aligned}
T(J) & =\frac{\left(N^{2} \rho-2 s\right)(s+\rho)+N s(2 s-\rho)}{N[N(s+\rho)-3 s]} \\
\Delta(J) & =\frac{s(\rho+s)[2 s-N(N-1)(\rho+s)]}{N[N(s+\rho)-3 s]}
\end{aligned}
$$

Now note that $N \geq 3$ suffices to ensure that (i) the denominator of $\Delta(J)$ is positive, and (ii) the numerator of $\Delta(J)$ is negative. Therefore, $\Delta(J)<0$ for all $N \geq 3$; as a result, the pair $\left(p^{s s}, q^{s s}\right)$ identifies a saddle point. 
Otherwise, if $N \in\{1,2\}$, one has to take into account the sign of $T(J)$, $\Delta(J)$ and $[T(J)]^{2}-4 \Delta(J)$ together:

- $N=1$. In this case,

$$
\begin{gathered}
T(J)=\rho>0, \\
\Delta(J)=\frac{2 s \rho(s+\rho)}{2 s-\rho} \lessgtr 0 \forall s \lessgtr \frac{\rho}{2}, \\
{[T(J)]^{2}-4 \Delta(J) \gtrless 0 \forall s \lessgtr \frac{\rho}{2} .}
\end{gathered}
$$

Hence, in the monopoly case $\left(p^{s s}, q^{s s}\right)$ is (i) a saddle point for all $s<$ $\rho / 2$, (ii) an unstable focus for all $s>\rho / 2$.

- $N=2$. In this case,

$$
\begin{gathered}
T(J)=\frac{s^{2}+2 \rho^{2}}{2 s-\rho} \gtrless 0 \forall s \lessgtr 2 \rho, \\
\Delta(J)=\frac{s(s+\rho)(2 s+3 \rho)}{s-2 \rho} \lessgtr 0 \forall s \lessgtr 2 \rho, \\
{[T(J)]^{2}-4 \Delta(J) \gtrless 0 \forall s \lessgtr 2.226 \rho .}
\end{gathered}
$$

Hence, in the duopoly case $\left(p^{s s}, q^{s s}\right)$ is (i) a saddle point for all $s<2 \rho$, (ii) a stable node for all $s \in(2 \rho, 2.226 \rho)$; a stable focus for all $s>$ $2.226 \rho$.

This concludes the proof. 


\section{References}

[1] Caputo, M.R. (2007), "The Envelope Theorem for Locally Differentiable Nash Equilibria of Finite Horizon Differential Games", Games and Economic Behavior, forthcoming.

[2] Cellini, R. and L. Lambertini (2004), "Dynamic Oligopoly with Sticky Prices: Closed-Loop, Feedback and Open-Loop Solutions", Journal of Dynamical and Control Systems, 10, 303-14.

[3] Dixit, A.K. (1986), "Comparative Statics for Oligopoly", International Economic Review, 27, 107-22.

[4] Dockner, E.J, S. Jørgensen, N.V. Long and G. Sorger (2000), Differential Games in Economics and Management Science, Cambridge, Cambridge University Press.

[5] Fershtman, C. and M.I. Kamien (1987), "Dynamic Duopolistic Competition with Sticky Prices", Econometrica, 55, 1151-64.

[6] Fershtman, C. and M.I. Kamien (1990), "Turnpike Properties in a Finite-Horizon Differential Game: Dynamic Duopoly with Sticky Prices", International Economic Review, 31, 49-60.

[7] Friedman, J.W. (1977), Oligopoly and the Theory of Games, Amsterdam, North-Holland.

[8] Mehlmann, A. (1988), Applied Differential Games, New York, Plenum Press. 
[9] Simaan, M. and T. Takayama (1978), "Game Theory Applied to Dynamic Duopoly Problems with Production Constraints", Automatica, 14, 161-66.

[10] Tsutsui, S. and K. Mino (1990), "Nonlinear Strategies in Dynamic Duopolistic Competition with Sticky Prices", Journal of Economic Theory, 52, 136-61. 